Document downloaded from:

http://hdl.handle.net/10251/148516

This paper must be cited as:

Velikova, N.; Mas Font, N.; Miguel-Romero, L.; Polo, L.; Stolte, E.; Zaccaria, E.; Cao, R.... (2017). Broadening the antibacterial spectrum of histidine kinase autophosphorylation inhibitors via the use of epsilon-poly-L-lysine capped mesoporous silica-based nanoparticles. Nanomedicine Nanotechnology Biology and Medicine. 13(2):569-581. https://doi.org/10.1016/j.nano.2016.09.011

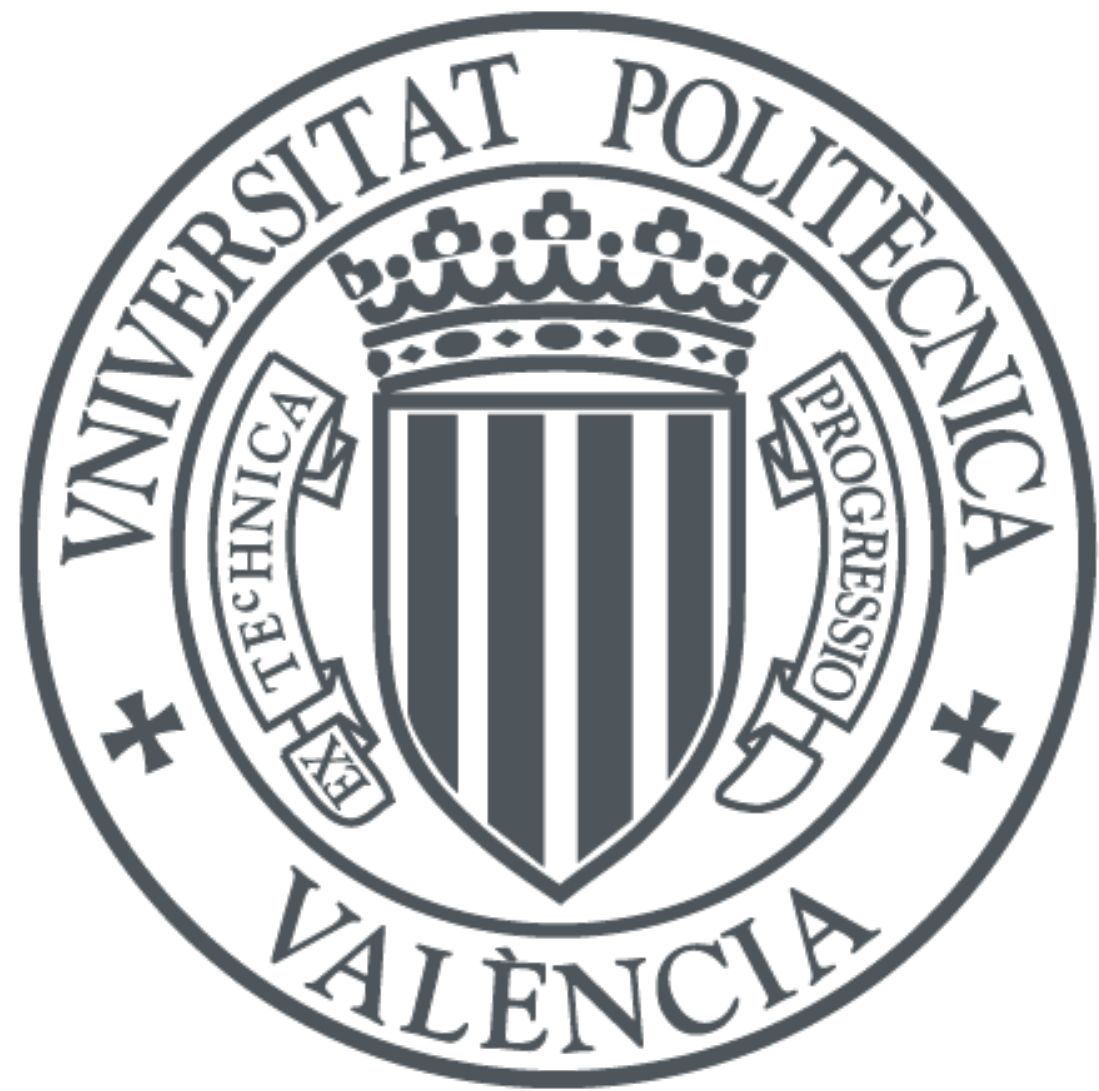

The final publication is available at

https://doi.org/10.1016/j.nano.2016.09.011

Copyright Elsevier

Additional Information 


\section{BROADENING THE ANTIBACTERIAL SPECTRUM OF HISTIDINE KINASE AUTOPHOSPHORYLATION INHIBITORS VIA THE USE OF E- POLY-L-LYSINE CAPPED MESOPOROUS SILICA-BASED NANOPARTICLES}

Nadya Velikova, 2, \#, Nuria Mas ${ }^{3,4}$, Laura Miguel-Romero², Lorena Polo Aguado ${ }^{3,4}$, Ellen Stolte$^{2}$, Edoardo Zaccaria ${ }^{2}$, Rui $\mathrm{Cao}^{2}$, Nico Taverne², José Ramón Murguía ${ }^{3}$, , Ramon Martinez-Manez ${ }^{3,4}$, Alberto Marina ${ }^{2,5}$, Jerry Wells ${ }^{2}$

${ }^{1}$ Instituto de Biomedicina de Valencia, Consejo Superior de Investigaciones Científicas (CSIC), Jaume Roig 11, 46010 Valencia, Spain

${ }^{2}$ Host-Microbe Interactomics Chair Group, Animal Sciences, University of Wageningen, P.O. Box 338, 6700 AH Wageningen, The Netherlands

${ }^{3}$ Instituto Interuniversitario de Investigación de Reconocimiento Molecular y Desarrollo Tecnológico (IDM). Unidad Mixta Universitat Politècnica de València-Universitat de València. Departamento de Quimica, Univesidad Politecnica de Valencia, Camino de Vera s/n, 46022 Valencia, Spain

${ }^{4}$ CIBER de Bioingeneria, Biomateriales y Nanomedicina, (CIBERBBN)

${ }^{5}$ CIBER de Enfermedades Raras (CIBERER), ISCIII, Valencia, Spain

Corresponding author \#

Nadya Velikova PhD

Address: Zodiac 122, De Elst 1, 6708WD, Wageningen, The Netherlands, Phone number: +31 317486041

E-mail address: nadya.velikova@wur.nl

This work was funded by FP7 ITN STARS - Scientific Training in Antimicrobial Research Strategies (Contract No. PITN-GA-2009-238490, J.M.W., A.M.), H2020 MSCA IF (AND-659121, N.V.), grant BIO2013-42619-P from the Ministerio de Economia y Competitividad (A.M.), grant from the Spanish Government (Project MAT2015-64139-C4-1-R, N.M., J.R.M, R.M.M.), and a grant from Generalitat Valenciana (Project PROMETEOII/2014/047, N.M.).

The authors declare no conflict of interests

ABSTRACT WORD COUNT 150

COMPLETE MANUSCRIPT WORD COUNT 4983

NUMBER OF REFERENCES 56

NUMBERF OF FIGURES 8 
NUMBER OF TABLES 4 


\section{ABSTRACT}

Two-component systems (TCS) regulate diverse processes such as virulence, stress responses, metabolism and antibiotic resistance in bacteria but are absent in humans, making them promising targets for novel antibacterials. By incorporating recently described TCS histidine kinase autophosphorylation inhibitors (HKAIs) into $\varepsilon$-poly-L-lysine capped nanoparticles (NPs) we could overcome the Gram negative (Gr-) permeability barrier for the HKAIs. The observed bactericidal activity against Gr- bacteria was shown to be due to the enhanced delivery and internalization of the HKAIs and not an inhibitory or synergistic effect of the NPs. The NPs had no adverse effects on mammalian cell viability or the immune function of macrophages in vitro and showed no signs of toxicity to zebrafish larvae in vivo. These results show that HKAIs are promising antibacterials for both Gr- and Gr+ pathogens and that NPs are a safe drug delivery technology that can enhance the selectivity and efficacy of HKAIs against bacteria.

\section{KEYWORDS}

Multi-drug resistance, Gram negative, nanotechnology, drug delivery, two-component systems

GRAPHICAL ABSTRACT: FIGURE 2 


\section{BACKGROUND}

Infections caused by Gram negative (Gr-) multidrug-resistant (MDR) bacteria have become a growing challenge worldwide (1). The most important resistance problems are encountered in Enterobacteriaceae, Pseudomonas aeruginosa, and Acinetobacter spp. which have developed resistance to last-line antibiotics such as expanded-spectrum cephalosporins and/ or carbapenems (2). Besides, urinary tract infections (UTIs) caused by Gruropathogenic Escherichia coli (UPEC) are among the most common bacterial infections in humans and recently multi-drug resistant E. coli have been reported (3), (4), (5). For this, novel strategies to target infections caused by Gr- bacteria are urgently needed.

Bacterial two-component systems (TCS) are signal transduction systems found in nearly all bacteria and have been proposed as promising antibacterial drug targets for both Gram positive $\left(\mathrm{Gr}^{+}\right)$and Gr- infections (6), (7), (8). A prototypical TCS consist of membrane bound histidine kinase (HK) and its cognate response regulator (RR) (9). Upon environmental stimuli the catalytic and ATP-binding (CA) domain of the HK autophosphorylates a conserved histidine found within the dimerization and histidine phosphotransfer (DHp) domain, which subsequently serves as the phosphodonor for a cognate RR (9). The changes in the phosphorylation levels of the RR are usually related to changes of the expression of target genes involved in the regulation of variety of processes including growth, virulence, antibacterial resistance and adaptation to environmental changes (6), (10), (11). Following structure-based and fragment-based drug discovery approaches (12), we have identified a set of bacterial histidine kinase autophosphorylation inhibitors (HKAIs) (Figure 1). These HKAIs inhibit the autophosphorylation of HKs from both Gr+ and Gr- bacteria, including YycG, belonging to the essential TCS YycFG found in many Gr+ pathogens and E. coli PhoR, belonging to the PhoRB TCS, which regulates phosphate 
homeostasis, adaptive stress responses and bacterial virulence (13), (14), (15), (16), (17). The HKAIs are bactericidal against a panel of $\mathrm{Gr}^{+}$, including methicillin-resistant Staphylococcus aureus (MRSA), with minimal bactericidal concentrations (MBC) of 8-16 $\mu \mathrm{g} / \mathrm{ml}$ (12). However, killing of Gr-, e.g. E. coli, was substantially less effective, presumably due to the composition of the lipopolysaccharide (LPS)-containing outer membrane (OM), which is known to hinder membrane permeability of hydrophobic antibiotics (18), (19). The low permeability OM barrier of Enterobacteriaceae is a major obstacle to the discovery and development of effective antibiotics against enteric Gr- pathogens. One strategy to overcome this problem is the use of cationic or amphiphilic molecules to facilitate permeability of the OM and sensitize Gr- bacteria to hydrophobic antibiotics (20), (21). Nanomedicines have also been successfully used to deliver therapeutic hydrophobic and hydrophilic drugs as well as proteins and peptides molecules and can be equipped with targeting molecules to deliver their cargo to specific organs or cells (22), (23), (24).

As delivery systems, mesoporous silica nanoparticles (MSNs) have attracted much attention due to their stability, biocompatibility, large load capacity and the possibility of easy functionalizing their surface to obtain targeting and drug release systems (25). The development of capped systems able to retain the payload yet releasing it upon the presence of a predefined stimulus has been proved to be an excellent approach to develop advanced nanodevices for controlled delivery applications. Although mesoporous silica supports have been used for the simple diffusion-controlled release of certain antibiotics (26), (27), (28), studies using capped materials to release MSN cargo on contact with bacteria have only recently been described (29). MSNs loaded with vancomycin, functionalized with n-[(3trimethoxysilyl)propyl]ethylendiamine $(f)$ and capped with a cationic polymer of $\varepsilon$-poly-Llysine (ePLL) showed an extended spectrum of activity against Gr- bacteria including E. coli (DH5a, 100, 405), Salmonella typhimurium and Erwinia carotovora (29). Most Gr- 
pathogens are intrinsically resistant to vancomycin due to its relatively high molecular weight and size and inability to pass through porins in the OM (30), but ePLL promoted ionic bonding of the nanoparticles loaded with vancomycin (N.Van) with the negatively charged $\mathrm{OM}$ and facilitated membrane permeability of vancomycin (31). Moreover when ePLL and vancomycin were used in the N.Van nano-formulation a potent synergistic effect was observed which did not occur when ePLL and vancomycin were used alone (29).

To test whether the approach described above could extend the spectrum of activity of the HKAIs to Gr- bacteria we loaded MSNs with different inhibitors, rhodamine as a control, and then capped the functionalised nanoparticles (NPs) with ePLL. The minimal inhibitory concentrations (MICs) of the NPs alone, NPs loaded with rhodamine and capped with ePLL (N.Rho), ePLL alone, and different HKAIs loaded to nanoparticles capped with ePLL were determined using a standard double dilution method and compared to the free HKAIs (12), (32), (33), (34). A further aim was to address concerns that these engineered NPs might also enhance cytotoxicity of the HKAIs for human and animal cells or have detrimental effects on the immune function of mononuclear cells that are crucial for innate defences against invading pathogens and initiation of adaptive responses. Lastly, we demonstrated that the NPs are not toxic to zebrafish larvae in vivo. 
METHODS

SYNTHESIS OF NANOPARTICLES

The NPs studied here were synthesized and characterized as previously described (29). Detailed information is provided in the Supplementary material.

\section{BACTERIAL STRAINS}

Escherichia coli CFT 073, E. coli DH5 $\alpha$ and S. marcescens 21639 were obtained from Leibniz Institute DSMZ - German Collections of Microorganisms and Cell Cultures (Braunschweig, Germany) and were propagated using standard microbiological procedures.

\section{ANTIMICROBIAL SUSCEPTIBILITY TESTING}

Minimal inhibitory concentrations (MICs) of MCM-41, NPs loaded with rhodamine and capped with $\varepsilon$-poly-L-lysine (N.Rho), ePLL alone, HKAIs loaded to nanoparticles capped with ePLL (N.HKAIs), and HKAIs alone (F.HKAIs) were determined using a standard double dilution method (32), (33), (34). Antimicrobial susceptibility testing was performed with inoculum of $1.10^{5}-1.10^{6} \mathrm{CFU} / \mathrm{ml}$. N.HKAIs were suspended in water and Mueller Hinton (MH) broth (1:1) to concentration corresponding to $1.10^{5} \mu \mathrm{g} / \mathrm{ml}$ (N.B2, N.B7,

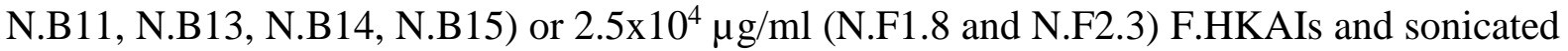
to obtain a homogenous suspension. The concentrations were chosen in relation to the $\mathrm{IC}_{50} \mathrm{~S}$ for HK autophosphorylation inhibition (micromolar range) and the MIC against $\mathrm{Gr}+(\leq 16$ 
$\mu \mathrm{g} / \mathrm{ml})(12)$. In the case of N.F1.8 and N.F2.3 lower concentration ranges were tested due to solubility issues. The suspensions were serially diluted in $\mathrm{MH}$ broth in 96-well plates. The MIC was recorded as the lowest concentration where no visible growth was observed. Minimal bactericidal concentrations (MBCs) were determined by plating $10 \mu \mathrm{l}$ of the wells where no growth was observed on $\mathrm{MH}$ agar plates and incubating overnight at $37{ }^{\circ} \mathrm{C}$ or by inoculating $90 \mu \mathrm{MH}$ broth with $10 \mu \mathrm{l}$ of the wells where no growth was observed. MBC was recorded as the lowest concentration where no colonies were formed or no visible growth was observed after overnight incubation at $37^{\circ} \mathrm{C}$.

\section{SYNERGY ASSESSMENT}

Synergy between $\varepsilon$-poly-L-lysine (ePLL) and F.HKAIs was assessed by the checkerboard method (35) in 96-well-plates in MH broth. Fractional inhibitory concentrations (FICs) were calculated based on the content in the wells in the growth-no growth area. FIC > 0.5 indicates no synergy (35).

\section{CELL LINES AND MEDIUM}

Caco-2 BBE cells (CRL 2102) and Raw 264.7 cells (Mouse leukemic monocyte macrophage cell line) were purchased from the American Type Culture Center (Manassas, VA) and grown in DMEM (Invitrogen, Paisley, UK) containing Glutamax, in RPMI 1640 (Invitrogen, Paisley, UK) supplemented with 2mM Glutamine, in DMEM, respectively.

Cells were grown in medium supplemented with $10 \%$ fetal bovine serum (FBS; PAA Laboratories, Colbe, Germany), $100 \mathrm{U} / \mathrm{ml}$ penicillin and $100 \mu \mathrm{g} / \mathrm{ml}$ streptomycin (Sigma, St. Louis, $\mathrm{MO}$ ) in an atmosphere of $5 \% \mathrm{CO}_{2}-95 \% \mathrm{O}_{2}$ at $37^{\circ} \mathrm{C}$ and were passaged weekly. 
Neutral-red (NR) uptake assay of cell viability with Caco-2 cells was performed as previously described (36). Briefly, after overnight incubation with the NPs (MCM-41, N.Rho and N.HKAIs), the F.HKAIs, or ePLL, $10 \mu \mathrm{l}$ of NR solution (33 $\mu \mathrm{g} / \mathrm{ml})$ was added to the wells. After $3 \mathrm{~h}$ of incubation at $37^{\circ} \mathrm{C}$, the medium was removed and cells were washed rapidly with $1 \mathrm{x}$ PBS. NR was extracted from the cells with $150 \mu 1 \%$ acetic acid-50\% ethanol, shaken for $10 \mathrm{~min}$ at RT. The NR content was measured on a SpectraMax M5 microplate reader (Molecular Devices) at $540 \mathrm{~nm}$. The readings were expressed as NR uptake relative to the uptake of the cells exposed to the negative control (medium or DMSO).

\section{ACTIVATION OF MACROPHAGES AND TREATMENT WITH NPS AND F.HKAIS}

Raw cells were seeded in 96-well-plates $\left(1.10^{5}\right.$ cells/well $)$ and allowed to attach to the bottom of the wells at $37^{\circ} \mathrm{C}$ and $5 \% \mathrm{CO}_{2}$ for $1 \mathrm{~h}$. Cells were treated with NPs (MCM-41, N.Rho, N.HKAIs) or F.HKAIs, in the presence or absence of $1 \mu \mathrm{g} / \mathrm{ml} \mathrm{LPS}$. Controls containing solvent (water or DMSO) were run in parallel. A nitric oxide (NO) assay and a viability assay (XTT assay) were run consecutively for every treatment group (see Supplementary Information). The macrophages cultures were incubated at $37^{\circ} \mathrm{C}$ and $5 \%$ $\mathrm{CO}_{2}$ for $24 \mathrm{~h}$ before performing the appropriate assays. 
Tumour-necrosis factor alpha $(\mathrm{TNF} \alpha)$ protein levels were measured by quantitative enzyme linked immunosorbet assays (ELISA) using the Mouse TNF alpha ELISA ReadySET-Go!® reagent set (Affymetrix, eBioscience, Netherlands). Briefly, after overnight incubation of Raw cells with NPs or F.HKAIs, before or after stimulation with $1 \mu \mathrm{g} / \mathrm{ml} \mathrm{LPS}$, plates were centrifuged at $2000 \mathrm{rpm}$, at RT for $7 \mathrm{~min}$. $75-\mu 1$ volumes of the supernatants were transferred to a new 96-well plate and ELISA was performed according to the manufactures protocol.

\section{ZEBRAFISH MAINTENANCE}

Zebrafish (Danio rerio) were bred at the animal facility of Wageningen University and Research Centrum as previously described (37), (38), (39), (37). The breeding tank was cleaned twice a day until hatching by removing debris and unfertilized eggs. Embryos were collected as eggs on different days and maintained in sterile oxygenated water at $28^{\circ} \mathrm{C}$. For experiments zebrafish embryos between 1 and 4 days post-hatching were used. After 4 days post-hatching, embryos were sacrificed by an overdose of the anesthetic 3-aminobenzoic acid (Tricaine, Sigma-Aldrich, USA) buffered with $1.5 \% \mathrm{NaHCO}_{3}$. All zebrafish were raised, maintained, and handled in compliance with the local animal welfare regulations, and according to standard protocols (zfin.org) under the guidelines of Wageningen University and Research Centre Institutional Animal Care and Use Committee.

\section{INCUBATION OF ZEBRAFISH LARVAE WITH N.RHO}

Zebrafish were cultured to 96-well plates (1 embryo/ well) in a total volume of $100 \mu \mathrm{l}$ oxygenated water at $28^{\circ} \mathrm{C}$. Then $1,2,5,10 \mu \mathrm{l}$ of the NP stock solution $(1 \mathrm{mg} / \mathrm{ml})$ were added 
to the wells. Each treatment group contained 5 larvae 3 days post-hatching and 5 replicates were tested per group. To visualize the NPs two embryos per group were anesthetized with $200 \mu \mathrm{g} / \mathrm{mL}$ 3-aminobenzoic acid. Images were taken after 1.5, 6 and 24 hours post incubation using Leica M205 FA Fluorescence Stereo Microscope (Leica Microsystems).

\section{CONFOCAL MICROSCOPY}

The DH5 $\alpha$ cells used for the confocal experiments were diluted 1/25 in MuellerHinton broth from an overnight culture and were grown with $1 \mathrm{mg} / \mathrm{ml}$ or $5 \mathrm{mg} / \mathrm{ml}$ of ePLLcapped NPs loaded with rhodamine (N.Rho) or empty nanoparticles supplemented with the same concentration of rhodamine (MCM41+Rho). After 4 hours the cells were imaged at 63 $\times$ HCXPL APO CS2 objetive (1.40 aperture) on a Confocal Leica TCS SP8 with a 561nm laser (567-700nm). The fluorescence cuantification was done with 5.2 zoom $(69,44 \mu \mathrm{m} x$ $69,44 \mu \mathrm{m})$, chosing only the cytoplasmatic portion of the cell, in ImageJ version Fiji program and the fluorescence was represented with GraphPad Prism. 
Results

SYNTHESIS AND CHARACTERIZATION OF MESOPOROUS SILICA-BASED NANOPARTICLES CAPPED WITH E-POLY-L-LYSINE AND LOADED WITH HKAIS

Mesoporous silica MCM-41 nanoparticles were loaded with HKAIs or rhodamine dye as a control and capped with $\varepsilon$-poly-L-lysine (N.HKAIs or N.Rho) as previously described (29). Figure 2 shows a schematic representation of the expected mechanism of action of N.Rho and N.HKAIs solids in the presence of Gr- bacteria. Standard procedures were used to characterize the prepared NP solids (see Supporting Information). Transmission electron microscopy (TEM) confirmed the mesoporous morphology of the MCM-41 NPs (diameter ca. $100 \mathrm{~nm}$ ) and in the final capped NPs (see Figure 3). Powder X-ray diffraction (PXRD), carried out on the starting MCM-41 NPs, showed the (100) low-angle reflection peak that is characteristic of these mesoporous materials. Preservation of the (100) reflection in the following solids (e.g. N.Rho) clearly confirmed that the loading and chemical modification processes did not damage the 3D mesoporous structure (see Supporting Information). Moreover, the $\mathrm{N}_{2}$ adsorption-desorption isotherms (see Supporting Information) were typical of a mesoporous material (MCM-41) and gated and filled mesoporous systems (N.Rho and N.HKAIs). Moreover, from thermogravimetric and elemental analyses the organic content of N.HKAIs and N.Rho was determined and is shown in Table 1. The content of the N.HKAIs was between 5 and $138 \mathrm{mg}$ HKAIs/g solid (0.02 to $0.45 \mathrm{mM} \mathrm{HKAI/} \mathrm{g} \mathrm{solid),} \mathrm{and} \mathrm{the} \mathrm{ePLL}$ and silica content for the N.HKAIs or N.Rho was between 91 and $220 \mathrm{mg}$ ePLL/ g solid (0.022 - $0.054 \mathrm{mmol}$ ePLL/g solid) and between 51 and 59\% silica, respectively. 
The antibacterial activities of N.HKAIs, F.HKAIs, ePLL, MCM-41 and N.Rho were evaluated by determining the optical density at $600 \mathrm{~nm}\left(\mathrm{OD}_{600}\right)$ of bacterial suspensions of $E$. coli CFT 073, E. coli DH5a, or S. marcescens 21639 incubated overnight (16 to 24 h) in 96well plates. The F.HKAIs did not inhibit bacterial growth at concentrations even as high as $500 \mu \mathrm{g} / \mathrm{ml}$ (MIC $>500 \mu \mathrm{g} / \mathrm{ml}$ ) with the exception of F.B14 and F.F1.8. F.B14 and F.F1.8, which had MICs of 500 and $250 \mu \mathrm{g} / \mathrm{ml}$ against E. coli CFT 073 and S. marcescens 21639 respectively (Table 2, (12)). The antibacterial activity of the N.HKAIs against E. coli CFT 073, E. coli DH5a, and S. marcescens 21639 was in all cases more than 10 times lower than the MICs of the F.HKAIs with MICs corresponding to 3 to $100 \mu \mathrm{g} / \mathrm{ml}$ HK inhibitors (Table 2). MICs corresponding to 3 to $100 \mu \mathrm{g} / \mathrm{ml} \mathrm{HK}$ inhibitors are comparable to the MICs of the F.HKAIs against $\mathrm{Gr}^{+}(12)$ supporting a common mechanism of action, i.e. HK inhibition.

\section{ANTIBACTERIAL ACTIVITY OF N.HKAIS IS DUE TO THE HK INHIBITORS}

To exclude the possibility that the observed antibacterial effect of the nanoformulations was due to the MCM-41 particles themselves or of the ePLL we tested the MIC of MCM-41 and N.Rho against E. coli CFT073 and S. marcescens (Table 3). MCM-41 and N.Rho did not inhibit bacterial growth, even at concentrations as high as $11 \mathrm{mg} / \mathrm{ml}$ (MIC $>11 \mathrm{mg} / \mathrm{ml}$ ) and $10 \mathrm{mg} / \mathrm{ml}$ (MIC > $10 \mathrm{mg} / \mathrm{ml}$ ), respectively. $10 \mathrm{mg} / \mathrm{ml} \mathrm{N}$.Rho corresponds to

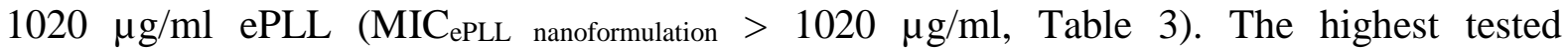
concentrations of MCM-41 and ePLL as part of N.Rho were higher than the MCM and ePLL content of the highest tested concentrations of the N.HKAIs. We also excluded the possibility that the observed antibacterial effect of the N.HKAIs was due to a synergistic effect of the HKAIs and ePLL using the checkerboard method with E. coli CFT 073 as the indicator 
strain. Using the same method, we also found no synergistic effect between ePLL and the free HK inhibitors (FICs > 0.5).

\section{EPITHELIAL CYTOTOXICITY OF NANOFORMULATIONS AND FREE HKAIS}

To be used as effective drugs, antibacterials should have high selectivity for the pathogen at the therapeutic concentrations reached in the body and not show toxicity to host cells. Although silica is generally considered to be non-cytotoxic, the ePLL-capped formulations of the silica-based NPs described here may affect their biocompatibility because of altered physicochemical properties or enhancement of HKAI toxicity to host cells. Therefore, to assess this we investigated the cytotoxicity of the studied NPs with and without incorporation of HKAIs.

Viability of human colon carcinoma cells (Caco-2) incubated with MCM-41, N.Rho, N.HKAIs, F.HKAIs, or ePLL was measured using the neutral-red (NR) uptake assay (36). The N.HKAIs were less cytotoxic than the F.HKAIs (Table 4, Figure S3), with IC50 values corresponding to $\geq 100 \mu \mathrm{g} / \mathrm{ml}$ of the HKAIs, whereas, the $\mathrm{IC}_{50}$ values of the F.HKAIs ranged from 3 to $134 \mu \mathrm{g} / \mathrm{ml}$. Thus the selectivity index (SI), i.e. mammalian cell cytotoxicity (IC50)/antibacterial effect (MIC), was improved for the N.HKAIs compared to the F.HKAIs (Table 4). Furthermore, MCM-41 and ePLL were not cytotoxic to Caco-2 cells at concentrations as high as $2742 \mu \mathrm{g} / \mathrm{ml}$ and $673 \mu \mathrm{g} / \mathrm{ml}$, respectively, corresponding to the highest content of MCM-41 and ePLL present in the N.HKAIs. N.Rho was also not cytotoxic at $1000 \mu \mathrm{g} / \mathrm{ml}$, corresponding to $102 \mu \mathrm{g} / \mathrm{ml}$ ePLL as part of the nanoformulation. 
The toxicity of the NPs capped with ePLL were also tested in vivo, using zebrafish larvae (40), (41), (42). Dispersion of N.Rho in the water containing the zebrafish embryos at concentrations of 10, 20 and $50 \mu \mathrm{g} / \mathrm{ml}$ did not result in any signs of toxicity or lethality to the larvae for up to 24 h hours (Figure 7). Nevertheless incubation with N.Rho led to a time and concentration dependent increase of rhodamine fluorescence in the intestine and in the yolk sac (Figure 7). This can be attributed to uptake of N.Rho via the gills or the skin (43), distribution in the blood and accumulation in the yolk sac and intestine. The gills are considered to be the main site of antigen uptake in fish, although entry through the skin has also been shown to play a significant role (43). If N.Rho was indeed taken up via the gills, it might have circulated through the vasculature to the yolk sac and the intestine. A similar distribution in the body was observed with liposomes delivered by immersion (44). Possible release of the rhodamine cargo due the encounter of the N.Rho with the yolk content or with Gr- negative bacteria normally present in the intestine may also be a factor influencing the observed results.

NPs AND NPs CAPPED WITH ePLL DO NOT AFFECT IMMUNE CELL FUNCTION

Immunotoxicity and altered immune responses are unwanted side effects precluding the clinical use of many drugs. To investigate potential for the NPs alone or N.HKAI formulation to elicit or attenuate inflammatory responses, we incubated them with macrophages for $24 \mathrm{~h}$ at $37^{\circ} \mathrm{C}$ with $5 \% \mathrm{CO}_{2}$ and measured NO production and secretion of pro-inflammatory cytokines such as tumour-necrosis factor alpha (TNF $\alpha)$.

$\mathrm{TNF} \alpha$ is involved in the process of inflammation and in cellular processes mediating protection to bacterial infections (45). Antibacterials ideally should not alter the level of TNF $\alpha$ production by activated and resting macrophages. Indeed, neither the NPs (MCM-41, 
N.Rho or N.B13) nor the HKAI F.B13 caused changes in TNF $\alpha$ secretion by Raw cells before or after stimulation with $1 \mu \mathrm{g} / \mathrm{ml}$ LPS (Figure 6).

The inducible high-output isoform of nitric oxide synthase (NOS2 or iNOS) lies at the interface between the innate and adaptive immune systems (46). Human NOS2 is most readily observed in monocytes or macrophages from patients with infectious or inflammatory diseases. Sustained production of NO endows macrophages with cytostatic or cytotoxic activity against viruses, bacteria, fungi, protozoa, helminths, and tumour cells. Other macrophage products such as acid, glutathione, cysteine, hydrogen peroxide, or superoxide enhance the antibacterial and cytotoxic actions of NO. To investigate whether the NPs and the HKAI F.B13 interfered with NO production by immune cells we incubated cultured macrophages with MCM-41, N.Rho, N.B13 and F.B13 with and without LPS $(1 \mu \mathrm{g} / \mathrm{ml})$ stimulation for 16 to $24 \mathrm{~h}$ and then measured NO production (Figure 4 and S4). Simultaneously, the effect of MCM-41, N.Rho, N.B13 and F.B13 on macrophage viability and TNF $\alpha$ protein levels were evaluated using the XTT assay and TNF $\alpha$ ELISA respectively (Figure 5, 6, and S4).

$1 \mathrm{mg} / \mathrm{ml} \mathrm{MCM-41} \mathrm{or} \mathrm{N.Rho} \mathrm{did} \mathrm{not} \mathrm{cause} \mathrm{significant} \mathrm{changes} \mathrm{in} \mathrm{NO} \mathrm{production} \mathrm{by}$ macrophages or in macrophage viability compared to the solvent control before or after stimulation with $1 \mu \mathrm{g} / \mathrm{ml}$ LPS. These results indicate that MCM-41 and N.Rho do not stimulate NO production by themselves and do not inhibit NO production induced by LPS. The production of NO before and after stimulation with $1 \mu \mathrm{g} / \mathrm{ml}$ LPS after incubation with N.B13 and F.B13 at concentrations corresponding to $100 \mu \mathrm{g} / \mathrm{ml}$ B13 was comparable to the solvent control. However, free HKAI F.B13 reduced viability of macrophages stimulated with LPS by more than 50\% at a concentration of $100 \mu \mathrm{g} / \mathrm{ml}$ and LPS stimulated macrophage viability by more than $50 \%$ at a concentration of $20 \mu \mathrm{g} / \mathrm{ml} \mathrm{F.B13.This} \mathrm{finding} \mathrm{suggests} \mathrm{that}$ F.B13 might induce an increase in NO production by macrophages but this is masked by its 
cytotoxic effect. The macrophage viability was decreased by N.B13 at concentrations corresponding to $100 \mu \mathrm{g} / \mathrm{ml}$ B13, implying that N.B13 also stimulates some NO production by macrophages. Nevertheless, these effects are observed at concentrations higher than the MIC of F.B13 for Gr+ bacteria and of N.B13 for Gr- bacteria (12) and Table 2, respectively). As the nanomaterials, MCM-41 and the control N.Rho did not cause significant changes in NO production or in macrophages viability, the N.B13 effect on NO production and macrophage viability can be attributed to release of the cargo, i.e. the HKAI F.B13. This is in agreement with the cell viability study of free HKAI F.B13 and Caco-2 cells (Table 4 and Figure S3) and indicates that the NPs and NPs capped with ePLL do not affect the function of innate immune cells.

ePLL-CAPPED NPS ENHANCE THE INTERNALIZATION OF THE CARGO

To study how the ePLL-capped NPs broaden the spectrum of the HKAIs in particular, and of vancomycin (29) we performed confocal microscopy experiments. Incubation of E. coli DH5 $\alpha$ with ePLL-capped NPs loaded with rhodamine resulted in concentration-dependent internalization of rhodamine (Figure 8). Incubation of E. coli DH5 $\alpha$ with MCM-41 and rhodamine separately and not as part of the nanoformulation did not result in internalization of rhodamine in the same experimental set up (Figure 8). These suggest that the ePLL-capped NPs enhance the internalization of the loaded content. 


\section{DISCUSSION}

The growing number of reports about the spread of multi-drug resistant (MDR) Grpathogens demands urgent development of novel approaches to combat the health risks associated with infections caused by Gr- bacteria (1). Due to low number of new classes of antibacterials entering the market over the past decade and growing incidence of infections due to MDR bacteria, effort is also being directed towards the improving pharmacological properties, potency and delivery of known antibiotics (21), (47), (29), (48), (22). Here we employed a recently described nanotechnology approach (29), to enhance permeability of recently identified putative histidine kinase autophosphorylation inhibitors (HKAIs) in Grbacteria and demonstrated that they are bactericidal for Gr- bacteria (12). The previously reported HKAIs were bactericidal for Gr+ bacteria but not Gr- bacteria despite the fact they inhibit the autophosphorylation of HKs from both Gr- and Gr+ in vitro (12). The Gr+ bacteria tested with these HKAIs all possess homologues of an essential TCS (YycFG), which may explain the antibacterial bactericidal effect of the inhibitors against Gr+. However, it was uncertain whether the lack of bactericidal activity for Gr- bacteria was due to low permeability of the OM or the fact that none of the individual TCS in Gr- are essential for viability under laboratory growth conditions.

The putative mechanism of action of the HKAIs is binding to the CA domain of HK and thus inhibiting ATP-binding and autophosphorylation of multiple HKs simultaneously. Therefore, in order to be active the HKAIs need to cross the bacterial cell envelope and reach the cytoplasm to exert their action. By incorporating the HKAIs into ePLL-capped NPs we could show that they were bactericidal to Gr- bacteria at MBC comparable to that shown for the free HKAIs against Gr+ pathogens (Table 2, (12)). As previously reported the HKAIs alone or the NPs themselves did not show antibacterial effect against Gr- (E. coli CFT073 or S. marcescens 21639, Table 3) (12), (29). Further antibacterial susceptibility testing in vitro and 
synergy testing of the components of the NPs revealed that the antibacterial effect of the N.HKAIs was solely dependent on the release of the NP cargo (i.e, the HKAIs) and not on the synergistic effect between the HKAIs and ePLL, or due to the presence of MCM-41 and/ or ePLL in the nanoformulations. These results indicate that HK autophosphorylation is a relevant target for antibacterials against Gr- provided the HKAIs overcome the Grpermeability barrier conferred by the LPS-containing OM. Furthermore, it indicates that simultaneous inhibition of multiple non-essential targets in Gr- bacteria leads to bactericidal effect.

Furthermore, an improved selectivity index ( $\mathrm{IC}_{50} / \mathrm{MIC}$ ) of the N.HKAIs compared to the F.HKAIs was observed (Table 4).This is an expected result because mesoporous NPs capped with cationic ePLL are designed to interact with the negatively charged OM of Grbacteria (29). Their proposed mechanism of action is attributed to ionic interaction of the positively charged ePLL capped NPs with the bacterial OM, followed by displacement and binding of ePLL to the Gr- cell membrane, thereby allowing the HKAIs to be released (29) (Figure 2). Incubation of E. coli DH5 $\alpha$ with the ePLL-capped NPs loaded with rhodamine, and a mixture of MCM-41 and rhodamine, showed that the nanoformulation enhances the internalization of the loaded content by E. coli DH5 $\alpha$ in a concentration-dependent manner (Figure 8). Additionally, the lack of synergistic effect between MCM-41 and the capping ePLL on cytotoxicity and immunotoxicty of the NPs, and the lack of toxicity to zebrafish embryos suggest that ePLL capped mesoporous NPs are safe to be used for antibacterial drug delivery.

The broadening of the antibacterial spectrum of the small-molecule HKAIs by ePLLcapped NPs suggests this approach has potential to be used to enhance the antibacterial spectrum of variety of antibiotics. 
A major challenge while translation will be to produce silica NPs with consistent physico-chemical characteristics at higher concentrations. One way to address this challenge is to optimize the large-scale synthesis of the NPs, or synthesize more batches of NPs as done so far $(23,24,29,49)$. In previous work and here we have shown that there is a good batchto-batch reproducibility in the physicho-chemical characteristics and biological activities of the synthesized NPs.

A further challenge while translation will also be to determine the route of administration. Most medicines can be administered by variety of routes. The choice of appropriate route of administration is determined based on the therapeutic objectives and on the properties of the drug. In the case of the ePLL-capped NPs loaded with HKAIs reported here the therapeutic objective would be to limit or eradicate bacterial infections caused by Grpathogens. Most common Gr- infections include recurrent urinary tract infections (UTIs), wound infections caused by $A$. baumanii and $P$. aeruginosa, respiratory infections by $P$. aeruginosa and other Gr- negatives, e.g. in lung disease in cystic fibrosis or nosocomial pneumonia, sexually transmitted infections by Neisseria gonorrhoea, bloodstream nosocomial infections by $K$. pneumoniae and P. aeruginosa, and others. To limit potential adverse effects, such as toxicity, interference with the host microbiota and inducing and spread of resistance, ideally the antibacterial NPs should be delivered locally to the site of infection (targeted drug delivery). In the case of UTIs, the bladder anatomy allows relatively easy and direct delivery of medicines through a catheter, allowing the drug to reach the target site with minimal side effects while avoiding first-pass metabolism. Other routes of administration applicable to medications for UTIs are reviewed in (50). For wound infections, direct delivery to the wound is the preferred option, reviewed in (51).

Infections of the respiratory tract are some of the most common causes of human illness and the leading cause of death from infectious diseases worldwide (52). Systemic 
delivery of antibacterials to treat lung infections face a number of obstacles. For example, it is well understood that effective antimicrobial therapy requires adequate drug concentrations at the target site of infection (53). To reach the deep airways in sufficient concentrations, high, often toxic doses of drugs would need to be given systemically. The inhalation of antibacterial agents on the other hand allows higher concentrations to be deposited directly in the lungs so that pathogens are exposed to supralethal concentrations while minimizing potential systemic toxicity by limiting absorption and avoiding unfavorable PK/PD consequences (54), (55). Furthermore, pulmonary drug delivery allows targeting the drug delivery directly to lung both for local and systemic treatment (56) making it a suitable route of administration for both respiratory and bloodstream infections.

SUMMARY

By combining two recently emerged strategies, loading of nanoparticles with antibacterials on the one hand, and targeting TCS signalling on the other hand, it was possible to achieve antibacterial bactericidal effect against Gr- bacteria in vitro with inhibitors of bacterial HK autophosphorylation. This shows that HKAIs are bactericidal to both Gr- and Gr+ presumably due to inhibition of multiple targets simultaneously. The NPs loaded with HKAIs did not show adverse effects on mammalian cell viability, or immunotoxicty in vitro. In fact, the N.HKAIs showed better SI compared to the F.HKAIs. Furthermore, the NPs did not cause adverse effects on zebrafish embryos. All together, these open promising possibilities for the treatment of Gr- infections and development of novel antibacterials and/ or broadening the spectrum of antibacterials using NPs.

\section{ACKNOWLEDGMENTS}


This work was funded by FP7 ITN STARS - Scientific Training in Antimicrobial Research Strategies (Contract No. PITN-GA-2009-238490, J.M.W., A.M.), H2020 MSCA IF (AND-659121, N.V.), grant BIO2013-42619-P from the Ministerio de Economia $y$ Competitividad (A.M.), grant from the Spanish Government (Project MAT2015-64139-C41-R, N.M., J.R.M, R.M.M.), and a grant from Generalitat Valenciana (Project PROMETEOII/2014/047, N.M.). 
Figure 1. Histidine kinase autophosphorylation inhibitors (HKAIs) Selected HKAIs inhibited the autophosphorylation of $E$. coli PhoR with $\mathrm{IC}_{50}$ ranging from 0.2 to $>2 \mathrm{mM}$. The HKAIs showed antibacterial effect for a panel of Gram-positive bacteria (12)with MICs in the range of 8 to $>500 \mu \mathrm{g} / \mathrm{ml}$. Only B14 and F1.8 showed antibacterial effect for Gram-negative bacteria with MICs $\geq 250$ or $\geq 125$, respectively. The $\mathrm{IC}_{50}$ (the concentration causing $50 \%$ reduction in autophosphorylation activity; mM) of each HKAIs for E. coli PhoR (PhoR IC50) is presented together with a consensus MIC (minimal inhibitory concentrations) in antimicrobial susceptibility testing assays with a panel of Gram-positive (Gr+) and Gramnegative (Gr-) bacteria.

Figure 2. Schematic representation and putative mechanism of action of the nanoparticles Nanometric mesoporous MCM-41 phase (ca. 100 nM; MCM-41) functionalized with n-[(3trimethoxysilyl)propyl]ethylendiamine triacetic acid trisodium salt (1; blue) was capped with $\varepsilon$-poly-L-lysine cationic polymer (ePLL; red rod) and loaded with histidine kinase autophosphorylation inhibitors (HKAIs, pink balls). Putative mechanism of action of the nanoparticles (NPs) is attributed to interaction of the positively charged capped NPs with the negatively charged Gr- bacterial cell wall and displacement of the capping ePLL which results in release of the loaded HKAIs inside the bacterium and subsequently to inhibition of two-component systems (TCS) signalling.

Figure 3. TEM image of the inorganic MCM-41 calcined matrix (A) and some of the final solids (B, C, D and E, correspond to N.Rho, NB.2, NB.14 and NF1.8 final solids, respectively). The images clearly show the mesoporous structure of the matrix which was maintained after capping with ePLL and loading with HKAIs.

Figure 4. Dose effects of MCM-41 (A), N.Rho (B), N.B13 (C) and F.B13 (D) on NO production by cultured macrophages (Raw cells). NO production is not affected by MCM-41 
(A), N.Rho (B), N.B13 (C), F.B13 (D) or the solvents (H2O and DMSO, E) with or without stimulation with $1 \mu \mathrm{g} / \mathrm{ml}$ LPS. The results are presented relative to the NO production induced by stimulation with $1 \mu \mathrm{g} / \mathrm{ml}$ LPS. The error bars present the SEM of at least three independent experiments in duplicate.

Figure 5. Dose effects of MCM-41 (A), N.Rho (B), N.B13 (C) and F.B13 (D) on cell viability of cultured macrophages (Raw cells). Cell viability is not affected by MCM-41 (A), N.Rho (B) or the solvents (H2O and DMSO, E) with or without stimulation with $1 \mu \mathrm{g} / \mathrm{ml}$ LPS. Reduction in cell viability by N.B13 (C) is observed only at the highest tested concentration (100 $\mu \mathrm{g} / \mathrm{ml}$ B13) with stimulation with $1 \mu \mathrm{g} / \mathrm{ml}$ LPS. Reduction of cell viability by F.B13 (D) is observed at the highest tested concentration (100 $\mu \mathrm{g} / \mathrm{ml}$ B13) with or without stimulation with $1 \mu \mathrm{g} / \mathrm{ml}$ LPS and at $20 \mu \mathrm{g} / \mathrm{ml}$ B13 with stimulation with $1 \mu \mathrm{g} / \mathrm{ml}$ LPS. The results are presented relative to the viability of the solvent controls (H2O or DMSO). The error bars present the SEM of at least two independent experiments in duplicate.

Figure 6. Dose effects of MCM-41 (A), N.Rho (B), N.B13 (C) and F.B13 (D) on TNFa levels of cultured macrophages (Raw cells). TNF $\alpha$ produced in Raw cells after incubation with MCM-41 (A), N.Rho (B), N.B13 (C), F.B13 (D) or by the solvents $\left(\mathrm{H}_{2} \mathrm{O}\right.$ and DMSO, E) with or without stimulation with $1 \mu \mathrm{g} / \mathrm{ml}$ LPS. The results are presented relative to the TNF $\alpha$ levels after stimulation with $1 \mu \mathrm{g} / \mathrm{ml}$ LPS. The error bars present the SEM of at two independent experiments in duplicate.

Figure 7. Incubation of zebrafish embryos with N.Rho. Rhodamine fluorescence in the yolk sack and the intestine is increased in concentration-dependent and time-dependent manner. Figure 8. Confocal imaging and quantification of NPs in DH5 $\alpha$ cells 
(A) Confocal images with 63x objective. Internalizaiton of rhodamine is only observed in E. coli CFT073 incubated with ePLL-capped NPs loaded with rhodamine and not in E. coli CFT073 incubated with a mixture of MCM-41 and rhodamine. The internalization of rhodamine is concentration-dependent. (B) Quantification of rhodamine fluorescence in E. coli DH5 $\alpha$ incubated for $4 \mathrm{~h}$ with 1 or $5 \mathrm{mg} / \mathrm{ml} \mathrm{N.Rho} \mathrm{and} \mathrm{empty} \mathrm{NPs} \mathrm{and} \mathrm{rhodamine}$ separately (MCM-41 and rhodamine) (20 different cells of each sample). 
References

1. Curcio D. Multidrug-Resistant Gram-Negative Bacterial Infections: Are you Ready for the Challenge? Current clinical pharmacology. 2014;9(1):27-38.

2. Dancer SJ. The problem with cephalosporins. The Journal of antimicrobial chemotherapy. 2001;48(4):463-78.

3. Ibrahim ME, Bilal NE, Hamid ME. Increased multi-drug resistant Escherichia coli from hospitals in Khartoum state, Sudan. African health sciences. 2012;12(3):368-75.

4. Alhashash F, Weston V, Diggle M, McNally A. Multidrug-resistant Escherichia coli bacteremia. Emerging infectious diseases. 2013;19(10):1699-701.

5. Vigil KJ, Adachi JA, Aboufaycal H, Hachem RY, Reitzel RA, Jiang Y, et al. Multidrug-resistant Escherichia coli bacteremia in cancer patients. American journal of infection control. 2009;37(9):7415.

6. Velikova N, Bem AE, van Baarlen P, Wells JM, Marina A. WalK, the Path towards New Antibacterials with Low Potential for Resistance Development. ACS Medicinal Chemistry Letters. 2013;4(10):891-4 .

7. Rutherford ST, Bassler BL. Bacterial quorum sensing: its role in virulence and possibilities for its control. Cold Spring Harbor perspectives in medicine. 2012;2(11).

8. Rasko DA, Moreira CG, Li de R, Reading NC, Ritchie JM, Waldor MK, et al. Targeting QseC signaling and virulence for antibiotic development. Science. 2008;321(5892):1078-80.

9. Casino P, Rubio V, Marina A. The mechanism of signal transduction by two-component systems. Current opinion in structural biology. 2010;20(6):763-71.

10. Skerker JM, Prasol MS, Perchuk BS, Biondi EG, Laub MT. Two-component signal transduction pathways regulating growth and cell cycle progression in a bacterium: a system-level analysis. PLoS biology. 2005;3(10):e334.

11. Wick LM, Egli T. Molecular components of physiological stress responses in Escherichia coli. Advances in biochemical engineering/biotechnology. 2004;89:1-45.

12. Velikova N, Fulle S, Manso AS, Mechkarska M, Finn P, Conlon JM, et al. Putative histidine kinase inhibitors with antibacterial effect against multi-drug resistant clinical isolates identified by in vitro and in silico screens. Scientific reports. 2016;6:26085.

13. Velikova N, Bem AE, van Baarlen P, Wells JM, Marina A. WalK, the Path towards New Antibacterials with Low Potential for Resistance Development. ACS medicinal chemistry letters. 2013;4(10):891-4.

14. Crepin S, Chekabab SM, Le Bihan G, Bertrand N, Dozois CM, Harel J. The Pho regulon and the pathogenesis of Escherichia coli. Veterinary microbiology. 2011;153(1-2):82-8.

15. Chekabab SM, Harel J, Dozois CM. Interplay between genetic regulation of phosphate homeostasis and bacterial virulence. Virulence. 2014;5(5).

16. Chekabab SM, Jubelin G, Dozois CM, Harel J. PhoB activates Escherichia coli O157:H7 virulence factors in response to inorganic phosphate limitation. PloS one. 2014;9(4):e94285.

17. Chekabab SM, Jubelin G, Dozois CM, Harel J. PhoB activates Escherichia coli O157:H7 virulence factors in response to inorganic phosphate limitation. PloS one. 2014;9(4).

18. Nikaido $\mathrm{H}$. Molecular basis of bacterial outer membrane permeability revisited. Microbiology and molecular biology reviews : MMBR. 2003;67(4):593-656.

19. Page MG. The role of the outer membrane of Gram-negative bacteria in antibiotic resistance: Ajax' shield or Achilles' heel? Handbook of experimental pharmacology. 2012(211):67-86. 20. Brehm-Stecher BF, Johnson EA. Sensitization of Staphylococcus aureus and Escherichia coli to antibiotics by the sesquiterpenoids nerolidol, farnesol, bisabolol, and apritone. Antimicrobial agents and chemotherapy. 2003;47(10):3357-60.

21. Bolla JM, Alibert-Franco S, Handzlik J, Chevalier J, Mahamoud A, Boyer G, et al. Strategies for bypassing the membrane barrier in multidrug resistant Gram-negative bacteria. FEBS letters.

2011;585(11):1682-90. 
22. Huh AJ, Kwon YJ. "Nanoantibiotics": a new paradigm for treating infectious diseases using nanomaterials in the antibiotics resistant era. Journal of controlled release : official journal of the Controlled Release Society. 2011;156(2):128-45.

23. Aznar E, Oroval M, Pascual L, Murguia JR, Martinez-Manez R, Sancenon F. Gated Materials for On-Command Release of Guest Molecules. Chemical reviews. 2016;116(2):561-718.

24. Aznar E, Martinez-Manez R, Sancenon F. Controlled release using mesoporous materials containing gate-like scaffoldings. Expert opinion on drug delivery. 2009;6(6):643-55.

25. Sancenón F, Pascual L, Oroval M, Aznar E, Martínez-Máñez R. Gated Silica Mesoporous Materials in Sensing Applications. ChemistryOpen. 2015;4(4):418-37.

26. Lai CY, Trewyn BG, Jeftinija DM, Jeftinija K, Xu S, Jeftinija S, et al. A mesoporous silica nanosphere-based carrier system with chemically removable CdS nanoparticle caps for stimuliresponsive controlled release of neurotransmitters and drug molecules. Journal of the American Chemical Society. 2003;125(15):4451-9.

27. Doadrio AL, Doadrio JC, Sánchez-Montero JM, Salinas AJ, Vallet-Regí M. A rational explanation of the vancomycin release from SBA-15 and its derivative by molecular modelling. Microporous and Mesoporous Materials. 2010;132(3):559-66.

28. Molina-Manso D, Manzano M, Doadrio JC, Del Prado G, Ortiz-Pérez A, Vallet-Regí M, et al. Usefulness of SBA-15 mesoporous ceramics as a delivery system for vancomycin, rifampicin and linezolid: a preliminary report. International journal of antimicrobial agents. 2012;40(3):252-6. 29. Mas N, Galiana I, Mondragon L, Aznar E, Climent E, Cabedo N, et al. Enhanced efficacy and broadening of antibacterial action of drugs via the use of capped mesoporous nanoparticles. Chemistry. 2013;19(34):11167-71.

30. Waltho JP, Williams DH. The natural design of vancomycin family antibiotics to bind their target peptides. Ciba Foundation symposium. 1991;158:73-86; discussion 7-91, 2-7.

31. Ye R, Xu H, Wan C, Peng S, Wang $L, X u H$, et al. Antibacterial activity and mechanism of action of epsilon-poly-L-lysine. Biochemical and biophysical research communications.

2013;439(1):148-53.

32. Conlon JM, Mechkarska M, Prajeep M, Sonnevend A, Coquet L, Leprince J, et al. Hostdefense peptides in skin secretions of the tetraploid frog Silurana epitropicalis with potent activity against methicillin-resistant Staphylococcus aureus (MRSA). Peptides. 2012;37(1):113-9.

33. Clinical Laboratory and Standards Institute Methods for Antimicrobial Susceptibility Testing of Anaerobic Bacteria; Approved Standard. M11-A7. 7th ed.2007.

34. CLSI. Methods for dilution antimicrobial susceptibility tests for bacteria that grow aerobically. Wayne, PA: CLSI; 2009.

35. Gialdroni Grassi G, Ferrara A, Peona V, Colombo ML. [Evaluation of the bactericidal activity of the combination of fosfomycin with other antibiotics by 2 different methods (checkboard and bactericidal curves)]. Giornale italiano di chemioterapia. 1983;30(2-3):77-84.

36. Repetto G, del Peso A, Zurita JL. Neutral red uptake assay for the estimation of cell viability/cytotoxicity. Nature protocols. 2008;3(7):1125-31.

37. Zaccaria E, Cao R, Wells JM, van Baarlen P. A Zebrafish Larval Model to Assess Virulence of Porcine Streptococcus suis Strains. PloS one. 2016;11(3):e0151623.

38. Kessels MY, Huitema LF, Boeren S, Kranenbarg S, Schulte-Merker S, van Leeuwen JL, et al. Proteomics analysis of the zebrafish skeletal extracellular matrix. PloS one. 2014;9(3):e90568.

39. Fiaz AW, Leon-Kloosterziel KM, Gort G, Schulte-Merker S, van Leeuwen JL, Kranenbarg S. Swim-training changes the spatio-temporal dynamics of skeletogenesis in zebrafish larvae (Danio rerio). PloS one. 2012;7(4):e34072.

40. van der Sar AM, Musters RJ, van Eeden FJ, Appelmelk BJ, Vandenbroucke-Grauls CM, Bitter W. Zebrafish embryos as a model host for the real time analysis of Salmonella typhimurium infections. Cellular microbiology. 2003;5(9):601-11.

41. Meijer AH, Spaink HP. Host-pathogen interactions made transparent with the zebrafish model. Current drug targets. 2011;12(7):1000-17. 
42. Medina C, Royo JL. Zebrafish as a model organism to study host-pathogen interactions. Methods. 2013;62(3):241-5.

43. Nakanishi T, Ototake M. Antigen uptake and immune responses after immersion vaccination. Developments in biological standardization. 1997;90:59-68.

44. Ruyra A, Cano-Sarabia M, Garcia-Valtanen P, Yero D, Gibert I, Mackenzie SA, et al. Targeting and stimulation of the zebrafish (Danio rerio) innate immune system with LPS/dsRNA-loaded nanoliposomes. Vaccine. 2014;32(31):3955-62.

45. Cooper AM, Appelberg R, Orme IM. Immunopathogenesis of Mycobacterium avium infection. Frontiers in bioscience : a journal and virtual library. 1998;3:e141-8.

46. MacMicking J, Xie QW, Nathan C. Nitric oxide and macrophage function. Annual review of immunology. 1997; 15:323-50.

47. O'Shea R, Moser HE. Physicochemical properties of antibacterial compounds: implications for drug discovery. Journal of medicinal chemistry. 2008;51(10):2871-8.

48. Abed N, Couvreur P. Nanocarriers for antibiotics: A promising solution to treat intracellular bacterial infections. International journal of antimicrobial agents. 2014;43(6):485-96.

49. Sancenon F, Pascual L, Oroval M, Aznar E, Martinez-Manez R. Gated Silica Mesoporous Materials in Sensing Applications. ChemistryOpen. 2015;4(4):418-37.

50. Zacche MM, Srikrishna S, Cardozo L. Novel targeted bladder drug-delivery systems: a review. Research and reports in urology. 2015;7:169-78.

51. Boateng JS, Matthews KH, Stevens HN, Eccleston GM. Wound healing dressings and drug delivery systems: a review. Journal of pharmaceutical sciences. 2008;97(8):2892-923.

52. Xu J, Murphy SL, Kochanek KD, Bastian BA. Deaths: Final Data for 2013. National vital statistics reports : from the Centers for Disease Control and Prevention, National Center for Health Statistics, National Vital Statistics System. 2016;64(2):1-119.

53. Leekha $S$, Terrell CL, Edson RS. General principles of antimicrobial therapy. Mayo Clinic proceedings. 2011;86(2):156-67.

54. Flume PA, VanDevanter DR. Clinical applications of pulmonary delivery of antibiotics. Adv Drug Deliv Rev. 2015;85:1-6.

55. Restrepo MI, Keyt H, Reyes LF. Aerosolized Antibiotics. Respiratory care. 2015;60(6):762-1; discussion 71-3.

56. Patil JS, Sarasija S. Pulmonary drug delivery strategies: A concise, systematic review. Lung India : official organ of Indian Chest Society. 2012;29(1):44-9. 